\title{
Internal validation of risk models in lung resection surgery: Bootstrap versus training-and-test sampling
}

Alessandro Brunelli, MD, and Gaetano Rocco, MD ${ }^{\mathrm{b}, *}$

Objective: The objective of the present analysis was to compare the performance of a lung resection mortality model developed by means of logistic regression and bootstrap analysis with that of multiple mortality models developed by using the traditional training-and-test method from the same dataset.

Methods: Eleven mortality models (1 developed by means of logistic regression and bootstrap validation and the other 10 developed by means of the traditional trainingand-test random splitting of the dataset) were generated by the data of unit A (571 patients submitted to major lung resection). The performances of each of the 11 mortality models were then evaluated by assessing the distribution of the respective c-statistics in 1000 bootstrap samples derived from unit B (224 patients).

Results: The first model (logistic regression and bootstrap analysis) had good discrimination among the 1000 bootstrap external samples (c-statistics $>0.7$ in $80 \%$ of samples and $>0.8$ in $38 \%$ of samples). Among the 10 training-and-test models, only one model had a similar performance, whereas the others had a poorer discrimination.

Conclusions: The traditional training-and-test method for risk model building proved to be unreliable across multiple external populations and was generally inferior to bootstrap analysis for variable selection in regression analysis. Therefore the use of bootstrap analysis must be recommended for every future model-building process.

From the Unit of Thoracic Surgery, "Umberto I" Regional Hospital, Ancona, Italy, and the Division of Thoracic Surgery, ${ }^{\mathrm{b}}$ Sheffield Teaching Hospital, Sheffield, United Kingdom.

Received for publication Nov 29, 2005; revisions received Jan 3, 2006; accepted for publication Feb 6, 2006.

Address for reprints: Alessandro Brunelli, MD, Via S. Margherita 23, Ancona 60129, Italy (E-mail: alexit_2000@yahoo.com).

*Dr Rocco is currently affiliated with the Division of Thoracic Surgery, National Cancer Institute, Pascale Foundation, Naples, Italy.

J Thorac Cardiovasc Surg 2006;131:1243-47 $0022-5223 / \$ 32.00$

Copyright $(\odot) 2006$ by The American Association for Thoracic Surgery

doi:10.1016/j.jtcvs.2006.02.002
$\mathrm{R}$ isk stratification and outcome analysis can be used to judge effectiveness of care and to assist providers in quality improvement activities, such as cost containment, patients' education, effectiveness of care studies, and improvement in provider practices.

Regression analyses are the analytic techniques most commonly used for risk modeling. However, the resultant models are useful only if they reliably predict outcomes for patients by determining significant risk factors associated with the outcome of interest. A problem might arise from this dependence on risk factor analysis. Different investigators evaluating the same predictors through regression analysis might obtain heterogeneous results because of methodologic discrepancies and inadvertent biases introduced in the statistical elaboration. ${ }^{1}$

In the early 1980s, computer-intensive computational techniques, termed bootstrap methods, were popularized. ${ }^{2-6}$ Bootstrap analysis is a simulation method for statistical inference, which, if applied to regression analysis, can provide variables that have a high degree of reproducibility and reliability as independent risk factors of the given outcome.

In fact, the predictive validity of a model can be assessed not only in one randomly split set of patients, as in the traditional training-and-test method, but also in perhaps hundreds or, typically, 1000 new different samples of the same number of patients as the original database obtained by means of resampling with replacement.

We hypothesized that the traditional training-and-test method for model building might generate models that are heavily biased by the characteristics of the patients 


\author{
Abbreviations and Acronyms \\ $\mathrm{CAD}=$ coronary artery disease \\ DLCO = carbon monoxide lung diffusion capacity \\ $\mathrm{FEV}_{1}=$ forced expiratory volume in 1 second \\ ppoDLCo $=$ predicted postoperative carbon monoxide \\ lung diffusion capacity \\ $\operatorname{ppoFEV}_{1}=$ predicted postoperative forced expiratory \\ volume in 1 second
}

who are sampled to derive and test them. The external performance of these types of models could be extremely variable and therefore totally unreliable. On the other hand, by using the entire dataset for model construction and bootstrap analysis for validation and variable selection, a more robust and stable model would be obtained, which can be more reliably applied to external patients.

Therefore the objective of the present study was to compare the performance of a mortality model adjusted for the covariates contributing to the risk of death developed from the entire dataset of patients submitted to major lung resection in one single unit and validated by using the bootstrap procedure with that of multiple mortality models developed by using the training-and-test method from the same dataset. To this purpose, each model was assessed in 1000 external bootstrap samples derived from another set of patients operated on in another unit during the same period.

\section{Patients and Methods}

A population of 571 patients undergoing major lung resection (479 lobectomies-bilobectomies and 92 pneumonectomies) from January 2000 through December 2004 in a thoracic surgery unit (unit A) was used to develop mortality models. Mortality was considered as that occurring within 30 days from the operation or over a longer period if the patient was still hospitalized.

Two different model-building approaches were used. The first method (model A) consisted of using the entire dataset for model construction. The following variables were initially evaluated for possible association with postoperative mortality: age, body mass index (in kilograms per square meter), type of operation (lobectomy vs pneumonectomy), type of disease (benign vs malignant), neoadjuvant chemotherapy, presence of coronary artery disease (CAD), forced expiratory volume in 1 second $\left(\mathrm{FEV}_{1}\right)$, carbon monoxide lung diffusion capacity (DLCO), predicted postoperative $\mathrm{FEV}_{1}$ (ppoFEV ${ }_{1}$; calculated by using the formula),

\section{[Preoperative $F E V_{1} /$ Number of preoperative functioning segments $] \times$ Number of postoperative functioning segments}

and predicted postoperative DlCo (ppoDlco, calculated by using the formula).

\section{[Preoperative DLCO/Number of preoperative functioning segments $] \times$ Number of postoperative functioning segments}

The number of functioning segments was estimated by means of computed tomography, bronchoscopy, and quantitative perfusion lung scanning. Pulmonary function tests were performed according to the American Thoracic Society criteria. DLCo was measured by using the single-breath method. Results of spirometry were collected after bronchodilator administration and were expressed as a percentage of predicted value for age, sex, and height according to the European Community for Steel and Coal prediction equations. ${ }^{7}$

Survivors and nonsurvivors were initially compared by means of univariate analyses performed with the unpaired Student $t$ test or the Mann-Whitney test for numeric variables and the $\chi^{2}$ test or the Fisher exact test for categoric variables. Multicollinearity among variables was obviated by using only one variable (selected by means of bootstrap analysis) in a set of variables with a correlation coefficient greater than 0.5 in the regression analysis. Variables with a $P$ value of less than .1 at univariate analysis were used as independent variables in a stepwise logistic regression analysis (dependent variable of mortality). A $P$ value of less than .1 was selected for variable retention in the final regression model. The model was then validated by means of bootstrap analysis. In the bootstrap procedure 1000 samples of 571 patients were sampled with replacement. Stepwise logistic regression analysis was applied to every bootstrap sample. The stability of the final model was assessed by comparing the frequency of occurrence of the variables of the final model in the bootstrap samples. If the predictors occurred in more than $50 \%$ of the bootstrap models, they were judged to be reliable and were retained in the final model. ${ }^{8}$ Unreliable variables, if present, were removed from the final model.

The second method (model B) consisted of the traditional training-and-test splitting method. The dataset was randomly split into 2 sets of patients. The first set (60\% of the database) was used to develop the model. The same variables used in the first method were initially evaluated for possible association with postoperative mortality. Screening for univariate associations and multicollinearity was performed in the same way in the second method as described for the first method. Variables with a $P$ value of less than .1 at univariate analysis were used as independent variables in a stepwise logistic regression analysis (dependent variable of mortality). A $P$ value of less than .1 was selected for variable retention in the final regression model, for which the calibration and discrimination was assessed with the remaining $40 \%$ of patients (test set) by using the Hosmer-Lemeshow goodness-of-fit statistic and by using the c-statistics or area under the receiver operating characteristic curve. ${ }^{9-12}$ The proportion of patients sampled in the training and test samples (60\% and $40 \%$, respectively), was selected in accordance with recently published analyses on risk modeling in lung surgery. ${ }^{13}$

Ten models were developed by repeating the training-and-test method 10 times. Therefore a total of 11 mortality models were obtained. The performance of each of these models was assessed 1000 times, each time using a bootstrap sampling of 224 patients drawn with replacement from the database of unit B, by evaluating the distribution of the c-statistics in these samples. ${ }^{9-12}$

Prospective, electronic, quality-controlled, clinical databases at the 2 participating centers were used for the analysis of data. The study was approved by the local institutional review boards, and informed consent concerning prospective data collection was obtained from all patients. The authors had access to the primary 
TABLE 1. Characteristics of the patients in the 2 units used to derive (unit A) and externally validate (unit B) the 11 mortality models

\begin{tabular}{|c|c|c|c|}
\hline Variables & $\begin{array}{c}\text { Unit A } \\
\text { (571 patients) }\end{array}$ & $\begin{array}{c}\text { Unit B } \\
\text { (224 patients) }\end{array}$ & $P$ value \\
\hline Age & $67(9.6)$ & $61.3(12.2)$ & $<.0001^{*}$ \\
\hline Male sex, $\mathrm{n}(\%)$ & $450(79 \%)$ & $144(64 \%)$ & $<.0001 \dagger$ \\
\hline $\mathrm{BMI}, \mathrm{kg} / \mathrm{m}^{2}$ & $26.1(4.2)$ & $25.6(4.5)$ & $.12^{*}$ \\
\hline $\mathrm{FEV}_{1}, \%$ & $85.5(19.5)$ & $84(18.2)$ & $.3^{*}$ \\
\hline Dıco, \% & $76.6(18.7)$ & $81.4(18.2)$ & $.004^{*}$ \\
\hline ppoFEV $_{1}, \%$ & $67.4(17.1)$ & $62.4(17.8)$ & $.0004^{*}$ \\
\hline ppoDıco, \% & $60.4(16.6)$ & $60.6(16.6)$ & $.6^{*}$ \\
\hline Pneumonectomy, n (\%) & $92(16 \%)$ & $42(19 \%)$ & $.4 \dagger$ \\
\hline $\begin{array}{l}\text { Coronary artery disease, } \\
\mathrm{n}(\%)\end{array}$ & $70(12 \%)$ & $34(15 \%)$ & $.3 \dagger$ \\
\hline Malignant disease, n (\%) & $550(96 \%)$ & $185(83 \%)$ & $<.0001 \dagger$ \\
\hline $\begin{array}{l}\text { Neoadjuvant chemotherapy, } \\
\mathrm{n}(\%)\end{array}$ & $79(14 \%)$ & $25(11 \%)$ & $.3 \dagger$ \\
\hline Mortality, n (\%) & $25(4.4 \%)$ & $10(4.5 \%)$ & $.9 \dagger$ \\
\hline
\end{tabular}

Results are expressed as means \pm standard deviations unless otherwise specified. BMI, Body mass index; $F E V_{1}$, forced expiratory volume in 1 second; $D\left\llcorner C 0\right.$, carbon monoxide lung diffusion capacity; ppoFEV $_{1}$, predicted postoperative forced expiratory volume in 1 second; $p p o D\llcorner C O$, predicted postoperative carbon monoxide lung diffusion capacity. *Mann-Whitney test. $\dagger \chi^{2}$ test.

data, directed the analyses, and made all decisions pertaining to the article and its submission for publication.

All tests were 2-tailed and were entirely performed with the Stata 8.2 statistical software (Stata Corp, College Station, Tex).

\section{Results}

The characteristics of the patients in the 2 units analyzed in this study are shown in Table 1. The 2 populations differed in age, sex, type of disease, and pulmonary function parameters.

The first statistical method used to develop and validate the mortality model (by using bootstrap analysis) yielded the following regression equations:

$$
\begin{aligned}
& \text { Model A: InR/1 }+\operatorname{InR}=-6.3+0.09 * \\
& \text { Age }(\text { Bootstrap frequency, 86\%) - 0.048* } \\
& \text { ppoFEV } V_{1} \text { (Bootstrap frequency, 71\%) }
\end{aligned}
$$

(Hosmer-Lemeshow statistics, 7.2; $P=.5$; c-statistic, 0.76). The expression InR/1 + InR represents the probability of dying because in the logistic regression equation the logarithm of the odds of the outcome (termed the logit or $\log$ odds) is used as the dependent variable.

The second statistical method (training and test) repeated 10 times yielded the following different mortality models:

- Model B1: InR/1 + InR $=-0.66-0.05 *$ ppoDLCO

$+1.04 * C A D$ (test set: Hosmer-Lemeshow statistics, $5.9 ; P=.7 ;$ c-statistic, 0.67$)$.
TABLE 2. Distribution of different variables and their coefficients in the 11 regression mortality models

\begin{tabular}{lcccccc}
\hline Models & Intercept & Age & $\begin{array}{c}\text { ppoFEV }_{1}, \\
\%\end{array}$ & $\begin{array}{c}\text { ppoDıco, } \\
\%\end{array}$ & Pneumonectomy & CAD \\
\hline A & -6.3 & 0.09 & -0.048 & & & \\
B1 & -0.66 & & & -0.05 & & 1.04 \\
B2 & -3.355 & 0.056 & & -0.066 & & \\
B3 & -0.55 & & -0.06 & & & \\
B4 & -10.1 & 0.1 & & & 1.64 & \\
B5 & -8.26 & 0.108 & -0.05 & & & 1.79 \\
B6 & -10.2 & 0.096 & & & & 1.32 \\
B7 & -11.4 & 0.113 & & & 1.86 & \\
B8 & -10.9 & 0.108 & & & 1.45 & \\
B9 & -10.7 & 0.147 & -0.049 & & & 1.11 \\
B10 & 0.64 & & -0.067 & & & 1.62
\end{tabular}

ppoFEV ${ }_{1}$, Predicted postoperative forced expiratory volume in 1 second; ppoDıco, predicted postoperative carbon monoxide lung diffusion capacity; $C A D$, coronary artery disease.

- Model B2: InR/1 + InR $=-3.355+0.056 *$ Age $0.066 *$ ppoDLCO (test set: Hosmer-Lemeshow statistics, $18 ; P=.02$; c-statistic, 0.57$)$.

- Model B3: InR/1 + InR $=-0.55-0.06 *$ ppoFEV $V_{1}$ (test set: Hosmer-Lemeshow statistics, 14.2; $P=.07$; c-statistic, 0.61).

- Model B4: InR/1 + InR $=-10.1+0.1 *$ Age +1.64 * Pneumonectomy (test set: Hosmer-Lemeshow statistics, $8 ; P=.4 ; \mathrm{c}$-statistic, 0.65$)$.

- Model B5: $\operatorname{InR} / 1+\operatorname{InR}=-8.26+1.79 * C A D+$ $0.018 *$ Age $-0.05 *$ ppoFEV $_{1}$ (test set: HosmerLemeshow statistics, 25.5; $P=.001$; c-statistic, 0.67).

- Model B6: InR/1 + InR $=-10.2+1.32 * C A D+$ $0.096 *$ Age (test set: Hosmer-Lemeshow statistics, $21 ; P=.07$; c-statistic, 0.63).

- Model B7: InR/1 + InR $=-11.4+0.113 *$ Age + $1.86 *$ Pneumonectomy (test set: Hosmer-Lemeshow statistics, 21.6; $P=.06$; c-statistic, 0.64).

- Model B8: InR/1 + InR $=-10.9+0.108 *$ Age + $1.45 *$ Pneumonectomy (test set: Hosmer-Lemeshow statistics, $13.7 ; P=.09$; c-statistic, 0.64).

- Model B9: InR/1 + InR $=-10.7+0.147 *$ Age $0.049 *$ ppoFEV $1+1.11 * C A D$ (test set: HosmerLemeshow statistics, 23; $P=.03$; c-statistic, 0.68).

- Model B10: InR/1 + InR $=0.64+1.62 * C A D-$ $0.067 *$ ppoFEV $_{1}$ (test set: Hosmer-Lemeshow statistics, 23.8; $P=.02$; c-statistics, 0.59).

The distribution of the different predictors in the 11 mortality models is shown in Table 2 .

Table 3 and Figure 1 show the distribution of the c-statistics of each model in 1000 bootstrap samples derived from an external series of patients operated on in another unit (unit B) during the same period. Model A (regression plus bootstrap) had good discrimination among the bootstrap external samples, 
TABLE 3. Distribution of ROC areas of each model in 1000 bootstrap samples derived from unit $B$

\begin{tabular}{lcccc}
\hline Models & $\begin{array}{c}\text { \% ROC } \\
\text { area }>\mathbf{0 . 5}\end{array}$ & $\begin{array}{c}\text { \% ROC } \\
\text { area }>\mathbf{0 . 7}\end{array}$ & $\begin{array}{c}\text { \% ROC } \\
\text { area }>\mathbf{0 . 8}\end{array}$ & $\mathbf{9 5 \% ~ C L}$ \\
\hline Model A & 100 & 80 & 38 & $0.58-0.94$ \\
Model B1 & 80 & 11 & 0 & $0.4-0.79$ \\
Model B2 & 100 & 92 & 38 & $0.65-0.89$ \\
Model B3 & 89 & 31 & 9 & $0.37-0.86$ \\
Model B4 & 99 & 59 & 18 & $0.62-0.95$ \\
Model B5 & 100 & 51 & 7 & $0.56-0.86$ \\
Model B6 & 85 & 11 & 1 & $0.53-0.87$ \\
Model B7 & 99 & 59 & 15 & $0.62-0.95$ \\
Model B8 & 99 & 62 & 17 & $0.63-0.95$ \\
Model B9 & 99 & 64 & 17 & $0.58-0.9$ \\
Model B10 & 75 & 16 & 0 & $0.32-0.79$
\end{tabular}

Model $A$ is derived from the entire dataset of unit $A$ and validated by means of bootstrap bagging, and models B1 through B10 are derived from a randomly selected training set of unit $A(60 \%$ of patients) and validated in a test set of unit A (the remaining $40 \%$ of patients). ROC, Receiver operating characteristic; $C L$, confidence limit.

with a c-statistic greater than 0.7 in $80 \%$ of the samples and greater than 0.8 in $38 \%$ of the samples. Among the 10 trainingand-test models, only model B2 had a similar performance, whereas the other models had a much poorer discrimination.

\section{Discussion}

Risk modeling, which is the base of risk stratification, is essential for quality improvement activities in managed care systems. It serves multiple purposes, such as provider profiling, cost containment, patient counseling about the operative risk, planning of postoperative advanced care management, and construction of efficacy studies. The importance of risk models is such that their reliability and reproducibility in populations other than the one from which they were derived must be absolutely proved. Otherwise, any clinical and administrative decision based on these models can be critically flawed by their instability in external populations.

We hypothesized that the traditional training-and-test method for model building, consisting of a random splitting of the database into a derivation set from which to construct the model and a test set in which to assess its calibration and discrimination, might be subject to sampling noise. To this purpose, we repeated 10 training-and-test sessions, producing 10 corresponding mortality models. Seventy percent of these models included different combinations of variables. The performance of each of these models was assessed 1000 times, each time using a bootstrap sampling of 224 patients drawn from the dataset of another unit. The distribution of the c-statistics was extremely variable from one model to another, and in general, their performances in external samples were only modest. The development of risk-adjusted models by the method of training and testing appears therefore completely unreliable.

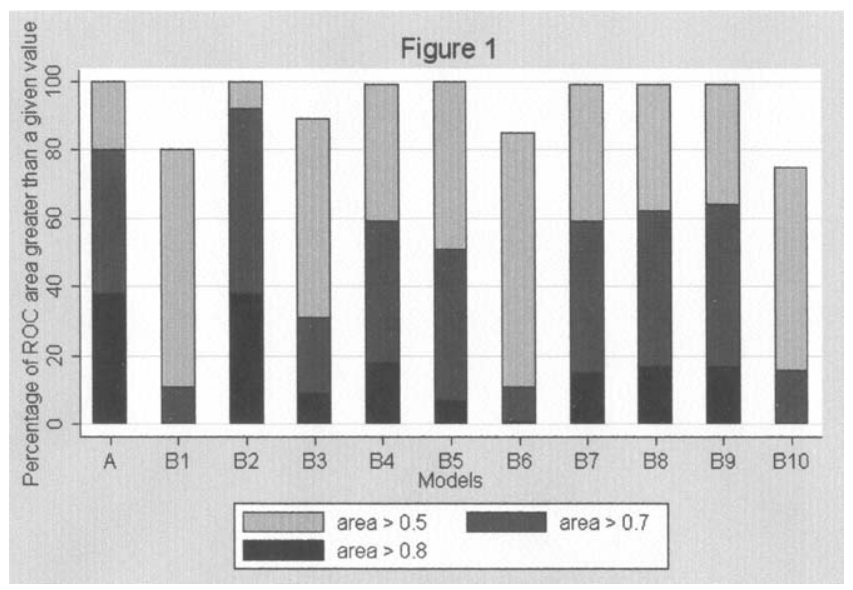

Figure 1. Distribution of the receiver operating characteristic $(R O C)$ areas of each model derived from unit $A$ in 1000 bootstrap samples drawn from unit $B$.

Bootstrap analysis was recently proposed as a breakthrough method for internal validation of surgical regression models. ${ }^{8,14}$ The main advantage of this technique is that the entire dataset can be used for model building, which would yield more robust models, especially in moderate-size databases and for rare outcomes (eg, mortality after major lung resection). ${ }^{15}$ Furthermore, the predictive validity of the model can be assessed not only in one randomly split set of patients but also typically in 1000 new different samples of the same number of patients as the original database obtained by means of resampling with replacement. By using this method, we constructed and validated a mortality model, which, when assessed in 1000 bootstrap samples drawn from another unit, performed better than the majority of the models developed by using the training-and-test method. This shows that the bootstrap procedure can yield stable models across multiple populations, warranting its use as a standard instrument in future model-building analyses. Yet a search of PubMed performed over the last 5 years yielded, at the time of this writing, only 16 surgical articles (published in the English literature and dealing with human subjects) that used logistic regression analysis and bootstrap for its validation $(0.003 \%$ of the total number of surgical articles that used logistic regression analysis and were published during the same period). It is clear that although bootstrap technology has broken down important barriers to surgical clinical research, ${ }^{8}$ its importance appears still largely underestimated by most surgeons. This might be due to the paucity of readily available high-quality statistical software incorporating this analysis or the lack of understanding of the methodology, which might make surgeons perceive this statistical technique itself as a barrier to their interpretation of clinical data analysis reports. In this regard 
a specific statistical training focusing on a reliable evaluation of the surgical outcome would be of help to disseminate a culture of quality improvement practice among surgeons.

On the basis of our results, we regard the process of developing risk models or risk factors without bootstrap validation as unreliable, obsolete, and resembling more an art than a science. ${ }^{8}$ In view of the unreliability of the training-and-test method, previously published reports using it should be interpreted with caution.

Bootstrap analysis can formalize the development of model building, removing much of the human biases associated with regression analysis, providing a balance between selecting risk factors that are not reliable (type I error) and overlooking variables that are reliable (type II error), and introducing a concrete measure of reliability of the risk factors. ${ }^{8}$ For this reason, the use of bootstrap analysis must be recommended for every future surgical modelbuilding process.

\section{References}

1. Naftel DC. Do different investigators sometimes produce different multivariable equations from the same data? J Thorac Cardiovasc Surg. 1994;107:1528-9.

2. Diaconis P, Efron B. Computer-intensive methods in statistics. Scientific American. 1983;248:96-109.
3. Efron B, Gong G. A leisurely look at the bootstrap, the jackknife and cross-validation. Am Stat. 1983;37:36-48.

4. Efron B, Tibshirani RJ. An introduction to the bootstrap. New York: Chapman \& Hall; 1993.

5. Breiman L. Bagging predictors. Machine Learning. 1996;26:123-40.

6. Efron B. The bootstrap and modern statistics. J Am Stat Assoc. 2000; 95:1293-6.

7. Quanjer PhH, Tammeling GJ, Cotes JE, Pedersen OF, Peslin R, Yernault JC. Lung volumes and forced ventilatory flows. Report Working Party. Standardization of lung function tests. European Community for Steel and Coal. Official statement of the European Respiratory Society. Eur Respir J. 1993;6(suppl 16):5-40.

8. Blackstone EH. Breaking down barriers: helpful breakthrough statistical methods you need to understand better. J Thorac Cardiovasc Surg. 2001;122:430-9.

9. Harrell FE, Lee KL, Califf RM, Pryor DB, Rosati RA. Regression modelling strategies for improved prognostic prediction. Stat Med. 1984;3:143-52.

10. Harrell FE, Lee KL, Mark DB. Multivariable prognostic models: issues in developing models, evaluating assumptions and adequacy, and measuring and reducing errors. Stat Med. 1996;15:361-87.

11. Hosmer DW, Lemeshow S. Applied logistic regression. New York: John Wiley \& Sons; 1989.

12. Grunkemeier GL, Jin R. Receiver operating characteristic curve analysis of clinical risk models. Ann Thorac Surg. 2001;72:323-6.

13. Berrisford R, Brunelli A, Rocco G, Treasure T, Utley M. The European Thoracic Surgery Database project: modelling the risk of inhospital death following lung resection. Eur J Cardiothorac Surg. 2005;28:306-11.

14. Grunkemeier GL, Wu Y. Bootstrap resampling methods: something for nothing? Ann Thorac Surg. 2004;77:1142-4.

15. Harrel FE. Regression modelling strategies with applications to linear models, logistic regression, and survival analysis. New York: SpringerVerlag; 2001. 